\title{
ON A CERTAIN GENERALISATION OF THE ITERATED FUNCTION SYSTEM
}

\author{
FILIP STROBIN ${ }^{凶}$ and JAROSŁAW SWACZYNA
}

(Received 26 January 2012; accepted 23 May 2012)

\begin{abstract}
We follow the idea of generalising the notion of classical iterated function systems, as presented by Mihail and Miculescu. We give their deliberations a more general setting and, using this general approach, study the generic aspect of the problem of existence of an attractor of a function system.
\end{abstract}

2010 Mathematics subject classification: primary 28A80; secondary 54E52.

Keywords and phrases: fractals, iterated function systems, fixed points, Baire category, porosity.

\section{Introduction}

The motivation for our study derives from the recent papers of Mihail [M] and Mihail and Miculascu [MM1, MM2], where the authors presented interesting generalisations of the well-known notion of iterated function systems (IFSs).

Originally, an IFS was a finite system of Banach contractions $\mathcal{S}:=\left(f_{1}, \ldots, f_{n}\right)$ of some complete metric space $X$. Such an IFS generates a natural mapping $F_{\mathcal{S}}: \mathbf{K}(X) \rightarrow$ $\mathbf{K}(X)$ :

$$
F_{\mathcal{S}}(D):=\bigcup_{k=1}^{n} f_{k}(D)
$$

$\left(\mathbf{K}(X)\right.$ is the space of all nonempty and compact subsets of $X$ ). The mapping $F_{\mathcal{S}}$ turns out to be a Banach contraction (we consider $\mathbf{K}(X)$ as a metric space with the Hausdorff metric $H$ ), so it has a unique fixed point, that is, a set $A(\mathcal{S}) \in \mathbf{K}(X)$ such that $F_{\mathcal{S}}(A(\mathcal{S}))=A(\mathcal{S})$.

Fixed points of IFSs are called attractors, or Hutchinson-Barnsley fractals. This nice idea (first presented by Hutchinson [H], then popularised by Barnsley [B]) attracted many mathematicians and yielded a plentiful crop of results. In particular, instead of selfmappings of a metric space $X$, Mihail and Miculescu [M, MM1, MM2] considered mappings from a Cartesian product $X \times \cdots \times X$ into $X$. They derived versions of fixed point theorems for such mappings and used them to obtain other ways

(c) 2012 Australian Mathematical Publishing Association Inc. 0004-9727/2012 \$16.00 
for generating fractals. (Note that fixed points of such mappings were also investigated earlier in the literature; see [Se].)

The fixed point theorems they generalised are the well-known Banach fixed point theorem (in [M, MM2]) and the Edelstein theorem which states that a selfmapping $f$ of a compact space, which satisfies $d(f(x), f(y))<d(x, y)$ for different $x, y$, also satisfies the conclusion of the Banach fixed point theorem (in [MM1]).

It is well known that both these famous fixed point results are special cases of the fixed point theorem for $\varphi$-contractions. Hence the question naturally arises whether it is possible to extend the considerations raised in [M, MM1, MM2] in this direction.

In this paper we give an affirmative answer to this question (Section 3). The proofs will follow the ideas presented in [M, MM1, MM2] but, since our setting is more general, some of them will be slightly shorter than the original ones. (It is often the case in mathematics that the more general the assumptions, the simpler the proofs are.)

Moreover, we study the generic aspects of the problem. In particular, we show that most nonexpansive function systems have attractors (Section 5).

\section{Notation and terminology}

In view of Section 5, we start by presenting some notions of porosity. Let $X$ be a metric space. In the following, $B(x, R)$ stands for an open ball centered at $x$ with radius $R>0$.

We say that $M \subset X$ is lower porous if

$$
\forall_{x \in M} \exists_{\alpha>0} \exists_{R_{0}>0} \forall_{R \in\left(0, R_{0}\right)} \exists_{z \in X} B(z, \alpha R) \subset B(x, R) \backslash M .
$$

If $M$ is a countable union of lower porous sets, then we say that $M$ is $\sigma$-lower porous. Clearly, $\sigma$-lower porosity implies meagreness, but the converse need not be true-in all 'reasonable' complete metric spaces there are sets which are meagre and are not $\sigma$-lower porous. Hence if we know that a particular set is not only meagre but also $\sigma$-lower porous, then we know that it is even smaller. In fact, there are many notions of porosity; for more information we refer the reader to survey papers [Z1, Z2].

Let $(X, d)$ and $(Y, \rho)$ be metric spaces. If $f: X \rightarrow Y$, then we denote by $\operatorname{Lip}(f)$ the Lipschitz constant of $f$ (in the following, $\inf \emptyset=\infty$ ):

$$
\operatorname{Lip}(f):=\inf \left\{c>0: \forall_{x, y \in X} \rho(f(x), f(y)) \leq c d(x, y)\right\} .
$$

If $\operatorname{Lip}(f) \leq 1$, then we say that $f$ is nonexpansive.

If for some nondecreasing, upper semicontinuous function $\varphi:[0, \infty) \rightarrow \mathbb{R}$ with $\varphi(t)<t$ for $t>0$,

$$
\forall_{x, y \in X} \rho(f(x), f(y)) \leq \varphi(d(x, y))
$$

then we say that $f$ is a generalised $\varphi$-contraction. Clearly, if $\operatorname{Lip}(f)<1$, then $f$ is also a generalised $\varphi$-contraction. (Simply set $\varphi(t):=t \operatorname{Lip}(f)$.) 
If $Y=X$, then every generalised $\varphi$-contraction is called a $\varphi$-contraction. Recall the following fixed point theorem. Part (i) is due to Browder [Br], and part (ii) is a consequence of [J, Theorem 11] (see also [J, Remark 11]).

THeOREM 2.1. Let $X$ be a complete metric space.

(i) If $f: X \rightarrow X$ is a $\varphi$-contraction, then $f$ satisfies the conclusion of the Banach fixed point theorem, that is, $f$ has a unique fixed point and each sequence of iterates of $f$ converges to that fixed point.

(ii) Let $\left(f_{n}\right)$ be a sequence of $\varphi$-contractions (for the same function $\varphi$ ) which is pointwise convergent to a function $f$ on $X$. Then $f$ is a $\varphi$-contraction. If also $\lim \inf _{t \rightarrow \infty}(t-\varphi(t))>0$, then the sequence of fixed points of the functions $f_{n}$ converges to the fixed point of $f$.

REMARK 2.2. In fact, we can slightly weaken the assumptions of part (ii). Namely, we can assume that $\left(f_{n}\right)$ converges to $f$ on a dense subset of $X$ and $f$ is continuous. Indeed, since $\operatorname{Lip}\left(f_{n}\right) \leq 1$, we instantly have that this implies that $\left(f_{n}\right)$ is pointwise convergent to $f$ (see also [MM1, Proposition 2.12]).

Remark 2.3. It is easy to see that if $X$ and $Y$ are compact and

$$
\forall_{x, y \in X} \rho(f(x), f(y))<d(x, y),
$$

then $f$ is a generalised $\varphi$-contraction. In particular, the Edelstein theorem follows from Theorem 2.1.

Recall that $\mathbf{K}(X)$ denotes the family of nonempty and compact subsets of $X$, considered as a metric space with a Hausdorff (or Hausdorff-Pompeiu) metric $H$ :

$$
\begin{aligned}
H(D, G) & :=\max \left\{\sup _{x \in D}\left(\inf _{y \in G} d(x, y)\right), \sup _{y \in G}\left(\inf _{x \in D} d(x, y)\right)\right\} \\
& =\inf \{r>0: D \subset G(r) \text { and } G \subset D(r)\},
\end{aligned}
$$

where $A(r):=\left\{x \in X: \exists_{y \in A} d(x, y)<r\right\}$.

It is well known that if $X$ is complete, then $\mathbf{K}(X)$ is also complete, and if $X$ is compact, then $\mathbf{K}(X)$ is also compact.

Now if $m \in \mathbb{N}$, then we denote by $X^{m}$ the Cartesian product of $m$ copies of $X$, endowed with the maximum metric:

$$
d_{\max }\left(\left(x_{1}, \ldots, x_{m}\right),\left(y_{1}, \ldots, y_{m}\right)\right):=\max \left\{d\left(x_{1}, y_{1}\right), \ldots, d\left(x_{m}, y_{m}\right)\right\},
$$

and if $m \in \mathbb{N}$ and $f_{1}, \ldots, f_{n}: X^{m} \rightarrow X$ are continuous functions and $\mathcal{S}:=\left(f_{k}\right)_{k=1}^{n}$, then we define $F_{\mathcal{S}}: \mathbf{K}(X)^{m} \rightarrow \mathbf{K}(X)$ in the following way:

$$
F_{\mathcal{S}}\left(D_{1}, \ldots, D_{m}\right):=\bigcup_{i=k}^{n} F_{f_{k}}\left(D_{1}, \ldots, D_{m}\right),
$$

where $F_{f}\left(D_{1}, \ldots, D_{m}\right):=f\left(D_{1} \times \cdots \times D_{m}\right):=\left\{f\left(x_{1}, \ldots, x_{m}\right): x_{i} \in D_{i}, i=1, \ldots, n\right\}$. We are ready to give the definition of a generalised iterated function system. 
Definition 2.4. Let $X$ be a metric space and $m, n \in \mathbb{N}$. Let $f_{1}, \ldots, f_{n}: X^{m} \rightarrow X$ be generalised $\varphi$-contractions. Then $\mathcal{S}:=\left(f_{i}\right)_{i=1}^{n}$ is called a generalised iterated function system (GIFS) of order $m$. If additionally $\operatorname{Lip}\left(f_{i}\right)<1$ for $i=1, \ldots, n$, then we call $\mathcal{S}$ a generalised classical iterated function system (GcIFS) of order $m$. If $\mathcal{S}$ is a GIFS, then the function $F_{\mathcal{S}}$, defined as in (2.2), is called the set function associated to $\mathcal{S}$.

REMARK 2.5. If every $f_{i}, i=1, \ldots, n$, is a generalised $\varphi$-contraction and satisfies (2.1) for a function $\varphi_{i}$, then it also satisfies (2.1) for $\varphi:=\max \left\{\varphi_{1}, \ldots, \varphi_{n}\right\}$, and $\varphi$ is nondecreasing, upper semicontinuous and $\varphi(t)<t$ for $t>0$. Hence if $\mathcal{S}=\left(f_{i}\right)_{i=1}^{n}$ is a GIFS, then there is one 'appropriate' function $\varphi$ such that each $f_{i}$ is a $\varphi$-contraction with this function $\varphi$. This observation will be important in the formulation of Theorem 3.7.

REMARK 2.6. Note that the GIFS considered in [MM2] (and the RIFS considered in $[\mathrm{M}]$ ) are precisely what we call GcIFS, and by Remark 2.3 , in the case when $X$ is compact, our definition of GIFS is equivalent to that given in [MM1]. Moreover, the case $m=1$ has also been considered in the literature (see [Ma]).

\section{Results}

We begin with a generalisation of Theorem 2.1, which can also be viewed as a generalisation of parts of [MM1, Theorem 3.4] and [M, Theorems 2.1 and 2.5].

Theorem 3.1. Let $(X, d)$ be a complete metric space and $f: X^{m} \rightarrow X$ be a function.

(i) If $f$ is a generalised $\varphi$-contraction, then there exists a unique $\alpha \in X$ such that $f(\alpha, \alpha, \ldots \alpha)=\alpha$ and for every $x_{1}, x_{2}, \ldots, x_{m} \in X$, the sequence $\left(x_{k}\right)_{k=1}^{\infty}$ defined by $x_{k+m}:=f\left(x_{k}, x_{k+1}, \ldots, x_{k+m-1}\right)$, for all $k \geq 1$, is convergent to $\alpha$.

(ii) If $\left(f_{n}\right)$ is a sequence of generalised $\varphi$-contractions (with the the same function $\varphi)$ which is pointwise convergent to $f$, then $f$ is a $\varphi$-contraction, and if $\left(\alpha_{n}\right)$ is such that $f_{n}\left(\alpha_{n}, \ldots, \alpha_{n}\right)=\alpha_{n}, n \in \mathbb{N}$, then $\alpha_{n} \rightarrow \alpha$, where $\alpha$ is such that $f(\alpha, \ldots, \alpha)=\alpha$.

REMARK 3.2. In fact, in part (i) we can replace pointwise convergence by pointwise convergence on a dense set, but we cannot skip the assumption that all $f_{n}$ are $\varphi$ contractions with the same function $\varphi$. An appropriate example can be found in [GD, p. 18]. (Note that [MM1, Theorem 3.4] is formulated for compact spaces.)

Remark 3.3. Note that [MM1, Theorem 3.4] and [M, Theorem 2.1] are formulated in slightly different (but equivalent) ways. Namely, the sequence $\left(x_{n}\right)$ is defined by $x_{m+k}:=f\left(x_{k+m-1}, \ldots, x_{k}\right)$ instead of $x_{m+k}:=f\left(x_{k}, \ldots, x_{m+k-1}\right)$. However, the proofs presented there are appropriate for the second case.

The proof of the theorem requires the following three lemmas. We skip the proof of the first lemma since it is mathematical folklore. 
Lemma 3.4. Let $X$ be a complete metric space and $f: X \rightarrow X$ be such that for some $p \in \mathbb{N}$, the function $f^{(p)}$ satisfies the hypothesis of the Banach fixed point theorem. Then $f$ satisfies the hypothesis of the Banach fixed point theorem.

Lemma 3.5. Let $(X, d)$ be a metric space, $m \in \mathbb{N}$ and $f: X^{m} \rightarrow X$ be nonexpansive. Define $h: X^{m} \rightarrow X^{m}$ by

$$
h\left(x_{1}, x_{2}, \ldots, x_{m}\right):=\left(x_{2}, \ldots, x_{m}, f\left(x_{1}, x_{2}, \ldots, x_{m}\right)\right) .
$$

Then $h$ is also nonexpansive.

Proof. Suppose that $f$ and $h$ are as in the formulation and $x=\left(x_{1}, \ldots, x_{m}\right)$, $y=\left(y_{1}, \ldots, y_{m}\right) \in X^{m}$. Then

$$
d(f(x), f(y)) \leq d_{\max }(x, y)=\max \left\{d\left(x_{1}, y_{1}\right), d\left(x_{2}, y_{2}\right) \ldots, d\left(x_{m}, y_{m}\right)\right\} .
$$

Hence

$$
\begin{aligned}
d_{\max }(x, y) & =\max \left\{d\left(x_{1}, y_{1}\right), d\left(x_{2}, y_{2}\right), \ldots, d\left(x_{m}, y_{m}\right)\right\} \\
& \geq \max \left\{d\left(x_{2}, y_{2}\right), d\left(x_{3}, y_{3}\right), \ldots, d\left(x_{m}, y_{m}\right), d(f(x), f(y))\right\} \\
& =d_{\max }(h(x), h(y)) .
\end{aligned}
$$

This completes the proof.

Lemma 3.6. Let $(X, d)$ be a metric space, $m \in \mathbb{N}$ and $x^{i}, y^{i} \in X^{m}, i=1, \ldots, m$. Assume that $f: X^{m} \rightarrow X$ is a generalised $\varphi$-contraction (with the function $\varphi$ ). Then

$$
d_{\max }\left(\left(f\left(x^{1}\right), \ldots, f\left(x^{m}\right)\right),\left(f\left(y^{1}\right), \ldots, f\left(y^{m}\right)\right)\right) \leq \varphi(\eta),
$$

where $\eta:=\max \left\{d_{\max }\left(x^{1}, y^{1}\right), \ldots, d_{\max }\left(x^{m}, y^{m}\right)\right\}$.

Proof. Let $i \in\{1, \ldots, m\}$. Then

$$
d\left(f\left(x^{i}\right), f\left(y^{i}\right)\right) \leq \varphi\left(d_{\max }\left(x^{i}, y^{i}\right)\right) \leq \varphi(\eta) .
$$

Hence

$$
d_{\max }\left(\left(f\left(x^{1}\right), \ldots, f\left(x^{m}\right)\right),\left(f\left(y^{1}\right), \ldots, f\left(y^{m}\right)\right)\right) \leq \varphi(\eta) .
$$

This completes the proof.

We now give the proof of Theorem 3.1.

Proof. We first prove (ii). Let $f_{n}, n \in \mathbb{N}$, be $\varphi$-contractions (with the same function $\varphi$ ) such that $\left(f_{n}\right)$ is pointwise convergent to $f$. Clearly, $f$ is a $\varphi$-contraction. Let $g, g_{n}: X \rightarrow X, n \in \mathbb{N}$, be the functions defined by $g(x)=f(x, x, \ldots, x),, g_{n}(x)=$ $f_{n}(x, x, \ldots, x), x \in X$. Clearly, $g_{n}$ are $\varphi$-contractions (with the same function $\varphi$ ) and the sequence $\left(g_{n}\right)$ is pointwise convergent to $g$. In particular, by Theorem 2.1, there exist unique $\alpha, \alpha_{n} \in X, n \in \mathbb{N}$, such that $\alpha=g(\alpha)=f(\alpha, \alpha, \ldots, \alpha), g_{n}\left(\alpha_{n}\right)=$ $f_{n}\left(\alpha_{n}, \alpha_{n}, \ldots, \alpha_{n}\right)=\alpha_{n}$ and $\alpha_{n} \rightarrow \alpha$. This concludes the proof of (ii). 
We now prove (i). Let $f$ be a generalised $\varphi$-contraction. As in the proof of (ii), it can be shown that there exists a unique $\alpha \in X$ such that $f(\alpha, \ldots, \alpha)=\alpha$. Now consider the function $h: X^{m} \rightarrow X^{m}$ given by

$$
h\left(x_{1}, x_{2}, \ldots, x_{m}\right):=\left(x_{2}, x_{3}, \ldots, x_{m}, f\left(x_{1}, x_{2}, \ldots, x_{m}\right)\right) .
$$

We will prove that $h^{(m)}$ is a $\varphi$-contraction. Let $x=\left(x_{1}, \ldots, x_{m}\right), y=\left(y_{1}, \ldots, y_{m}\right) \in X^{m}$ and set $\lambda:=d_{\max }(x, y)$. Define the points $x^{i}, y^{i} \in X^{m}, i=1, \ldots, m$, in the following way: $x^{1}:=x, y^{1}:=y$,

$$
x^{i+1}:=h\left(x^{i}\right)=\left(x_{i+1}, \ldots, x_{m}, f\left(x^{1}\right), \ldots, f\left(x^{i}\right)\right)
$$

and

$$
y^{i+1}:=h\left(y^{i}\right)=\left(y_{i+1}, \ldots, y_{m}, f\left(y^{1}\right), \ldots, f\left(y^{i}\right)\right) .
$$

By Lemma $3.5, h$ is nonexpansive, so $d_{\max }\left(x^{i}, y^{i}\right) \leq \lambda, i=1, \ldots, m$. Moreover, it can be easily seen that

$$
h^{(m)}(x)=h\left(x^{m}\right)=\left(f\left(x^{1}\right), \ldots, f\left(x^{m}\right)\right) \quad \text { and } \quad h^{(m)}(y)=h\left(y^{m}\right)=\left(f\left(y^{1}\right), \ldots, f\left(y^{m}\right)\right) .
$$

Hence, by Lemma 3.6,

$$
d_{\max }\left(h^{(m)}(x), h^{(m)}(y)\right) \leq \varphi(\lambda) .
$$

The above considerations show that for every $x, y \in X^{m}$,

$$
d_{\max }\left(h^{(m)}(x), h^{(m)}(y)\right) \leq \varphi\left(d_{\max }(x, y)\right),
$$

This shows that $h^{(m)}$ is a $\varphi$-contraction.

Hence $h^{(m)}$ fulfils the assumptions of Lemma 3.4 (taking $p=m$ ), so $h$ satisfies the hypotheses of the Banach fixed point theorem. That is, there exists $\left(\beta_{1}, \ldots, \beta_{m}\right) \in X^{m}$ such that $\left(\beta_{1}, \ldots, \beta_{m}\right)=h\left(\beta_{1}, \ldots, \beta_{m}\right)$ and every sequence of iterates of $h$ converges to $\left(\beta_{1}, \ldots, \beta_{m}\right)$.

In particular,

$$
\beta_{1}=\beta_{2}=\cdots=\beta_{m}=f\left(\beta_{1}, \beta_{2}, \ldots, \beta_{m}\right),
$$

and thus $\beta_{1}=\beta_{2}=\cdots=\beta_{m}=\alpha$. (Recall that $\alpha$ was a unique point for which $f(\alpha, \ldots, \alpha)=\alpha$.) Finally, for any $x_{1}, \ldots, x_{m} \in X$,

$$
(\alpha, \ldots, \alpha)=\left(\beta_{1}, \ldots, \beta_{m}\right)=\lim _{n \rightarrow \infty} h^{(n-1)}\left(x_{1}, \ldots, x_{m}\right)=\lim _{n \rightarrow \infty}\left(x_{n}, \ldots, x_{n+m-1}\right),
$$

so $x_{n} \rightarrow \alpha$ and the proof is complete.

We now prove a generalisation of [M, Lemma 2.4] and [MM1, Proposition 3.1].

THeorem 3.7. Let $(X, d)$ be a metric space, $m \in \mathbb{N}$ and $\mathcal{S}=\left(f_{i}\right)_{i=1}^{n}$ be a GIFS of order $m$ such that each $f_{i}$ is a $\varphi$-contraction with a function $\varphi$. Then the set function associated to $\mathcal{S}, F_{\mathcal{S}}$, is a generalised $\varphi$-contraction (with the same function $\varphi$ ). 
To prove the result we need some lemmas.

Lemma 3.8. Let $(X, d),(Y, \rho)$ be metric spaces and $f: X \rightarrow Y$ be a generalised $\varphi$ contraction. Then $F_{f}: \mathbf{K}(X) \rightarrow \mathbf{K}(Y)$ is also a generalised $\varphi$-contraction (with the same function $\varphi$ ), where

$$
\forall_{D \in \mathbf{K}(X)} F_{f}(D):=f(D) .
$$

Proof. Let $D, G \in \mathbf{K}(X)$. Set any $x \in D$ and let $\left(y_{n}\right) \subset G$ be such that $d\left(x, y_{n}\right) \rightarrow$ $\inf _{y \in G} d(x, y)$. Since $\varphi$ is upper semicontinuous and nondecreasing,

$$
\inf _{y \in G} \varphi(d(x, y)) \leq \limsup _{n \rightarrow \infty} \varphi\left(d\left(x, y_{n}\right)\right) \leq \varphi\left(\inf _{y \in G} d(x, y)\right) \leq \varphi(H(D, G)) .
$$

Hence

$$
\inf _{y \in G} \rho(f(x), f(y)) \leq \inf _{y \in G} \varphi(d(x, y)) \leq \varphi(H(D, G)) .
$$

Since $x$ was arbitrary,

$$
\sup _{x \in D}\left(\inf _{y \in G} \rho(f(x), f(y))\right) \leq \varphi(H(D, G)) .
$$

In the same way we show that

$$
\sup _{x \in G}\left(\inf _{y \in D} \rho(f(x), f(y))\right) \leq \varphi(H(D, G)),
$$

and the proof is complete.

Using the well-known fact that

$$
H\left(\bigcup_{i \in I} D_{i}, \bigcup_{i \in I} G_{i}\right) \leq \sup _{i \in I} H\left(D_{i}, G_{i}\right),
$$

we obtain the following corollary.

Corollary 3.9. Let $(X, d),(Y, \rho)$ be metric spaces, $n \in \mathbb{N}$ and $f_{1}, \ldots, f_{n}: X \rightarrow Y$. Define $F: \mathbf{K}(X) \rightarrow \mathbf{K}(Y)$ in the following way:

$$
F(D):=\bigcup_{k=1}^{n} f_{k}(D), \quad D \in \mathbf{K}(X) .
$$

If each $f_{i}$ is a generalised $\varphi$-contraction (with the same function $\varphi$ ), then $F$ is a generalised $\varphi$-contraction (with the function $\varphi$ ).

We now give the proof of Theorem 3.7.

Proof. It can be easily seen that if $X$ is a metric space and $m \in \mathbb{N}$, then, for every $D_{1}, \ldots, D_{m}, G_{1}, \ldots, G_{m} \in \mathbf{K}(X)$,

$$
\max \left\{H\left(D_{1}, G_{1}\right), \ldots, H\left(D_{m}, G_{m}\right)\right\}=H\left(D_{1} \times \cdots \times D_{m}, G_{1} \times \cdots \times G_{m}\right) .
$$


(It is important that we consider the maximum metric on the Cartesian product.) Moreover, for every $D_{1}, \ldots, D_{m} \in \mathbf{K}(X)$,

$$
F_{\mathcal{S}}\left(D_{1}, \ldots, D_{m}\right)=F\left(D_{1} \times \cdots \times D_{m}\right)
$$

where $F$ is defined as in Corollary 3.9. Hence the result follows from Corollary 3.9.

The next lemma can be compared with [MM1, Lemma 2.13]. We skip the proof since the result follows from [MM1, Lemma 2.11 and Proposition 2.12]. (In fact, we observe that in the formulation of [MM1, Lemma 2.11], we can replace the uniform convergence with uniform convergence on compact sets, and that $X$ can be any metric space.)

Lemma 3.10. Let $X$ be a metric space and $\mathcal{S}=\left(f_{1}, \ldots, f_{n}\right)$ be a GIFS. Let $\mathcal{S}_{k}=$ $\left(f_{1}^{k}, \ldots, f_{n}^{k}\right), k \in \mathbb{N}$, be a GIFS such that for any $i=1, \ldots, n,\left(f_{i}^{k}\right)$ is pointwise convergent to $f_{i}$ on a dense subset of $X$. Then $\left(F_{\mathcal{S}_{k}}\right)$ is pointwise convergent to $F_{\mathcal{S}}$.

We are ready to formulate our main result, which (in its main points-see Remark 3.2) is an extension of [MM1, Theorems 3.5 and 3.7] and [M, Theorems 2.2 and 2.6], and follows immediately from Theorems 3.1 and 3.7 and Lemma 3.10. Note that a similar observation to Remark 3.3 also holds here.

THEOREM 3.11. Let $X$ be a complete metric space and $\mathcal{S}=\left(f_{i}\right)_{i=1}^{n}$ be a GIFS of order $m$. Then there exists a unique $A(\mathcal{S}) \in \mathbf{K}(X)$ such that

$$
F_{\mathcal{S}}(A(\mathcal{S}), \ldots, A(\mathcal{S}))=A(\mathcal{S}) .
$$

Moreover, for any $H_{1}, \ldots, H_{m} \in \mathbf{K}(X)$, the sequence $\left(H_{k}\right)_{k=1}^{\infty}$, defined by

$$
H_{k+m}:=F_{\mathcal{S}}\left(H_{k}, \ldots, H_{k+m-1}\right), \quad k \in \mathbb{N},
$$

converges to $A(\mathcal{S})$.

Now let $\mathcal{S}_{k}=\left(f_{1}^{k}, \ldots, f_{n}^{k}\right), k \in \mathbb{N}$, be GIFS of order $m$ such that, for every $i=$ $1, \ldots, n,\left(f_{i}^{k}\right)$ converges pointwise to $f_{i}$ on a dense subset of $X$, and such that all $f_{i}^{k}$, $i=1, \ldots, n, k \in \mathbb{N}$, are $\varphi$-contractions (with the same function $\varphi$ ). Then $\left(A\left(\mathcal{S}_{k}\right)\right)_{k \in \mathbb{N}}$ converges to $A(\mathcal{S})$.

\section{Example}

We now present an example based on one presented in [MM2]. Throughout this section we assume that

$$
X=\left\{\left(x_{n}\right) \in c_{0}: \forall_{n \in \mathbb{N}} x_{n} \geq 0\right\},
$$

and we consider it as a metric subspace of $c_{0}$ (the Banach space of all real sequences which converge to zero). Note that $X$ is not compact, but remains complete. We begin with a definition. (Recall that classical IFSs are GIFSs of order one.) 
Definition 4.1. Let $k, m \in \mathbb{N}$ and let $\mathcal{S}=\left(f_{1}, \ldots, f_{k}\right)$ be a GIFS of order $m$, defined on the space $X$. We say that $\mathcal{S}$ is nice if each $f_{i}: X^{m} \rightarrow X$ is of the form

$$
f_{i}\left(\left(x_{n}^{1}\right), \ldots,\left(x_{n}^{m}\right)\right)=\left(f_{i}^{1}\left(x_{r_{1}^{i}}^{\epsilon_{1}^{i}}\right), f_{i}^{2}\left(x_{r_{2}^{i}}^{\epsilon_{2}^{i}}\right), f_{i}^{3}\left(x_{r_{3}^{i}}^{\epsilon_{3}^{i}}\right), \ldots\right)
$$

where $\left(r_{n}^{i}\right)_{n \in \mathbb{N}}$ is a sequence of naturals, and $\left(\epsilon_{n}^{i}\right)_{n \in \mathbb{N}}$ is a sequence of naturals from $\{1, \ldots, m\}$.

REMARK 4.2. Note that $\mathcal{S}=\left(f_{1}, \ldots, f_{k}\right)$ is nice if and only if each coordinate of each $f_{i}$ depends only on one (established) coordinate of $\left(x_{n}^{j}\right)$, where $j \in\{1, \ldots, m\}$ is also established.

Our main result in this section is the following theorem.

Theorem 4.3. There is a compact set $\emptyset \neq A \subset X$ such that:

(i) for some nice GIFS $\mathcal{S}, F_{\mathcal{S}}(A \times \cdots \times A)=A$;

(ii) for any IFS $\mathcal{S}^{\prime}$ which consists of Banach contractions, $F_{\mathcal{S}^{\prime}}(A) \neq A$;

(iii) for any nice IFS $\mathcal{S}^{\prime}$ which consists of $\varphi$-contractions, $F_{\mathcal{S}^{\prime}}(A) \neq A$;

(iv) for any nice GcIFS $\mathcal{S}^{\prime}, F_{\mathcal{S}^{\prime}}(A \times \cdots \times A) \neq A$.

The proof requires a number of lemmas.

Lemma 4.4. Let $0<c<a<d<b$ and $f:[a, b] \rightarrow[c, d]$ be linear with $f(a)=c$ and $f(b)=d$. For $x \in[d, b]$, set

$$
k_{x}=\max \left\{n \in \mathbb{N} \cup\{0\}: f^{n}(x) \geq a\right\}=\max \left\{n \in \mathbb{N}: f^{(n+1)}(x) \text { is defined }\right\} .
$$

Then

$$
\frac{\log \left(1-\frac{b-a}{b-d}(1-q)\right)}{\log q}-1 \leq k_{x}+1 \leq \frac{\log \left(1-\frac{b-a}{b-d}(1-q)\right)}{\log q}+1,
$$

where $q=(d-c) /(b-a)$.

Proof. The lemma (and its proof) become clearer if we make a cobweb graph of iterations of $f$ at a point $x$. Note that $f$ is given by a formula:

$$
\forall_{t \in[a, b]} f(t)=q t+p,
$$

where $p=(b c-d a) /(b-a)$.

Let $\left(a_{n}\right)_{n \geq 0}$ be the sequence of iterations of a linear extension $f$ on $b$ (in particular, $a_{0}=b$ and $a_{1}=f\left(a_{0}\right)=d$ ), and let $\left(b_{n}\right)_{n \geq 1}$ be defined by $b_{n}=a_{n-1}-a_{n}$ for $n \in \mathbb{N}$. For any $n \geq 2$,

$$
\begin{aligned}
b_{n} & =a_{n-1}-a_{n}=a_{n-1}-\left(q a_{n-1}+p\right)=a_{n-1}(1-q)-p \\
& =\left(q a_{n-2}+p\right)-a_{n-1} q-p=q\left(a_{n-2}-a_{n-1}\right)=q b_{n-1} .
\end{aligned}
$$

According to this, $\left(b_{n}\right)$ is a geometric sequence. 
Clearly, $k_{b}=k_{d}+1$ and, for any $x \in[d, b], k_{d} \leq k_{x} \leq k_{b}$. Hence it is enough to show that

$$
\frac{\log \left(1-\frac{b-a}{b-d}(1-q)\right)}{\log q}-1 \leq k_{b} \leq \frac{\log \left(1-\frac{b-a}{b-d}(1-q)\right)}{\log q} .
$$

By our assumptions, we can estimate

$$
b-a \leq \sum_{i=1}^{k_{b}+1} b_{i}=b_{1} \frac{1-q^{k_{b}+1}}{1-q}=(b-d) \frac{1-q^{k_{b}+1}}{1-q},
$$

which implies that

$$
\frac{\log \left(1-\frac{b-a}{b-d}(1-q)\right)}{\log q}-1 \leq k_{b}
$$

To get the second inequality in (4.1), we have to start with

$$
b-a \geq \sum_{i=1}^{k_{b}} b_{i}
$$

and proceed in an analogous way. Hence we get (4.1) and the proof is complete.

LEMma 4.5. There exists a function $\phi:[0, \infty) \rightarrow[0, \infty)$ with the following properties (in which $\left(\phi^{(n)}(1)\right)$ is the sequence of iterates of 1$)$ :

(a) $\phi$ is continuous, nondecreasing and $\phi(t)<t$ for $t>0$;

(b) $\phi$ is concave;

(c) $\forall_{m \geq 1}, \lim _{n \rightarrow \infty} \phi^{(m n)}(1) / \phi^{(n)}(1)=1$;

(d) $\forall_{n \geq 0}, \phi^{(n+1)}(1) / \phi^{(n)}(1) \geq \frac{1}{2}$.

Note that if $\phi:[0, \infty) \rightarrow[0, \infty)$ satisfies (a), then (c) is equivalent to:

(c') $\lim _{n \rightarrow \infty} \phi^{(2 n)}(1) / \phi^{(n)}(1)=1$.

Proof. Let $k \geq 2$ and define the function $\phi$ in the following way:

- $\phi(0)=0$;

- $\quad \phi(t)=1-(1 / k)$, for $t>1$;

- $\phi(1 / n)=1 / n-1 / k^{n}$, for $n \in \mathbb{N}$

- $\phi$ is linear on each interval $[1 /(n+1), 1 / n]$.

Note that we should write $\phi_{k}$ instead of $\phi$, since this function depends on $k$. However, our simplification will not lead to any confusion. For any $n \in \mathbb{N}$, set

$$
q_{n}=\frac{\phi\left(\frac{1}{n}\right)-\phi\left(\frac{1}{n+1}\right)}{\frac{1}{n}-\frac{1}{n+1}} .
$$

It is easy to see that, for each $n \in \mathbb{N}$,

$$
q_{n}=1-\frac{n(n+1)(k-1)}{k^{n+1}} .
$$

Step 1. We prove that, for $k \geq 3$, the function $\phi$ is concave. It is sufficient to prove that, for each $n \in \mathbb{N}, q_{n} \leq q_{n+1}$. Easy computations show that this is true for $k \geq 3$. 
Step 2. We prove that there exists $k_{0} \in \mathbb{N}$ such that, for $k \geq k_{0}$, the function $\phi$ satisfies

$$
\lim _{r \rightarrow \infty} \frac{\phi^{(2 r)}(1)}{\phi^{(r)}(1)}=1 .
$$

This will give us ( $\left.\mathrm{c}^{\prime}\right)$ and, in consequence, (c).

For every $n$, put

$$
A_{n}=\left\{r \in \mathbb{N}: f^{(r)}(1) \in\left[\frac{1}{n+1}, \frac{1}{n}\right]\right\} .
$$

It is enough to show that

$$
\forall_{n \in \mathbb{N}},\left|A_{n+1}\right| \geq 2\left|A_{n}\right|+1,
$$

where $|A|$ denotes the cardinality of $A$. Indeed, assume that we have proved (4.2). Then, by induction, we can easily show that

$$
\forall_{n \in \mathbb{N}},\left|A_{n+1}\right| \geq\left|A_{1} \cup \cdots \cup A_{n}\right|+1 .
$$

Now, for every $r \in \mathbb{N}$, let $n_{r}$ be such that $r \in A_{n_{r}}$. (If $r$ belongs to two sets, namely $r \in A_{n} \cup A_{n+1}$, then we set $n_{r}=n$.) Then $n_{r} \rightarrow \infty$. Moreover,

$$
\forall_{r \in \mathbb{N}}, n_{2 r}=n_{r} \text { or } n_{2 r}=n_{r}+1 \text {. }
$$

Indeed, by (4.3),

$$
\left|A_{n_{r}+1} \cup A_{n_{r}} \cup \cdots \cup A_{1}\right| \geq\left|A_{n_{r}+1}\right|+\left|A_{1} \cup \cdots \cup A_{n_{r}}\right|-1 \geq 2\left|A_{1} \cup \cdots \cup A_{n}\right|,
$$

which gives us (4.4). (We have used the fact that $\max \left(A_{1} \cup \cdots \cup A_{n}\right)=$ $\left|A_{1} \cup \cdots \cup A_{n}\right|$.) Finally, by (4.4),

$$
\forall_{r \in \mathbb{N}}, \frac{f^{(2 r)}(1)}{f^{(r)}(1)} \geq \frac{\frac{1}{n_{r}+2}}{\frac{1}{n_{r}}},
$$

which gives $f^{(2 r)}(1) / f^{(r)}(1) \rightarrow 1$.

We now show (4.2). It is easy to see that, for all $k \geq 3$ and $n \in \mathbb{N}$,

$$
0<\frac{1}{n+1}-\frac{1}{k^{n+1}}<\frac{1}{n+1}<\frac{1}{n}-\frac{1}{k^{n}}<\frac{1}{n} .
$$

Hence, by Lemma 4.4 , for every $n \in \mathbb{N}$,

$$
\frac{\log \left(\frac{1}{k}\right)}{\log \left(1-\frac{n(n+1)(k-1)}{k^{n+1}}\right)}-1 \leq\left|A_{n}\right| \leq \frac{\log \left(\frac{1}{k}\right)}{\log \left(1-\frac{n(n+1)(k-1)}{k^{n+1}}\right)}+1 .
$$

Then we only have to show that, for every $n \in \mathbb{N}$,

$$
2 \frac{\log \left(\frac{1}{k}\right)}{\log \left(1-\frac{n(n+1)(k-1)}{k^{n+1}}\right)}+3 \leq \frac{\log \left(\frac{1}{k}\right)}{\log \left(1-\frac{(n+1)(n+2)(k-1)}{k^{n+2}}\right)}-1 .
$$


It is easy to check that there exists $k_{0} \in \mathbb{N}$ such that, for any $k \geq k_{0}$ and $n \in \mathbb{N}$,

$$
\frac{\log \left(\frac{1}{k}\right)}{\log \left(1-\frac{n(n+1)(k-1)}{k^{n+1}}\right)} \geq 4 \text {. }
$$

Indeed, for $k \geq 3$, we have $q_{n} \leq q_{n+1}$, which shows that, for such $k$ and all $n \in \mathbb{N}$,

$$
\frac{\log \left(\frac{1}{k}\right)}{\log \left(1-\frac{n(n+1)(k-1)}{k^{n+1}}\right)} \geq \frac{\log \left(\frac{1}{k}\right)}{\log \left(1-\frac{2(k-1)}{k^{2}}\right)},
$$

so we only have to choose $k_{0} \in \mathbb{N}$ such that, for $k \geq k_{0}$,

$$
\frac{\log \left(\frac{1}{k}\right)}{\log \left(1-\frac{2(k-1)}{k^{2}}\right)} \geq 4 \text {. }
$$

Hence it is enough to show that there exists $k_{1} \in \mathbb{N}$ such that, for $k \geq k_{1}$ and all $n \in \mathbb{N}$,

$$
3 \frac{\log \left(\frac{1}{k}\right)}{\log \left(1-\frac{n(n+1)(k-1)}{k^{n+1}}\right)} \leq \frac{\log \left(\frac{1}{k}\right)}{\log \left(1-\frac{(n+1)(n+2)(k-1)}{k^{n+2}}\right)} .
$$

Again, by a standard but tedious computation, we can prove this. This concludes the proof of Step 2.

Step 3. We prove that there exists $k_{0} \in \mathbb{N}$ such that, for $k \geq k_{0}$ and every $a \in(0,1]$, $\phi(a) / a \geq \frac{1}{2}$. This will show (d).

For every $n \in \mathbb{N}$, put

$$
p_{n}=1-\frac{n(n+2)\left(k^{2}-1\right)}{2 k^{n+2}} .
$$

Then the linear mapping which connects points $\left(1 /(n+2), 1 /(n+2)-1 / k^{n+2}\right)$ and $\left(1 / n, 1 / n-1 / k^{n}\right)$ is given by

$$
\forall_{t \in[1 /(n+2), 1 / n]}, h_{n}(t)=p_{n} t+c_{n},
$$

where $c_{n}>0$ (if $k \geq 3$ ). We can show in a standard way that there exists $k_{0}$ such that for all $k \geq k_{0}$ and all $n \in \mathbb{N}, p_{n+1} \geq p_{n} \geq \frac{1}{2}$. Now let $k \geq k_{0}$ and choose any $a \in(0,1]$. Then $a \in[1 /(m+1), 1 / m]$ for some $m \in \mathbb{N}$. We then have

$$
\frac{\phi(a)}{a} \geq \frac{p_{m} a+c_{m}}{a} \geq p_{m}+\frac{c_{m}}{a} \geq \frac{1}{2} .
$$

This concludes the proof of Step 3.

Steps 1-3 imply Lemma 4.5.

Lemma 4.6. Assume that $I=I_{1} \times I_{2} \times \cdots=\prod_{n=1}^{\infty} I_{n}$ and $J=J_{1} \times J_{2} \times \cdots=\prod_{n=1}^{\infty} J_{n}$, where $I_{n}, J_{n} \subset[0, \infty)$ are closed bounded nontrivial intervals with $\operatorname{diam}\left(I_{n}\right) \rightarrow 0$, $\operatorname{diam}\left(J_{n}\right) \rightarrow 0, \quad I_{n+1} \subset I_{n}$ and $J_{n+1} \subset J_{n}$ for $n \in \mathbb{N}$. Let $f_{1}, f_{2}, \ldots:[0, \infty) \rightarrow[0, \infty)$ 
be $\varphi$-contractions (with the same function $\varphi$ ) satisfying

$$
\exists_{n_{0}} \forall_{n \geq n_{0}}, \quad \varphi\left(\operatorname{diam}\left(I_{n}\right)\right)<\operatorname{diam}\left(J_{n}\right) .
$$

Then for any sequence of naturals $\left(r_{n}\right)$, we have that if $f(I) \subset J$, then $f(I)$ is nowhere dense in $J$ (we consider $J$ as a subspace of $X$ ), where $f: X \rightarrow X$ is defined by $f\left(\left(x_{n}\right)\right)=\left(f_{1}\left(x_{r_{1}}\right), f_{2}\left(x_{r_{2}}\right), \ldots\right)$.

Proof. Note that the topologies on $I$ and $J$ are the same as the Tichonov topologies. In particular, $I$ and $J$ are compact.

Consider two cases.

Case 1. The sequence $\left(r_{n}\right)$ is one-to-one. Then, for infinitely many $n, r_{n} \geq n$. Indeed, assume for the sake of a contradiction that $r_{n} \geq n$ only for $n \in\left\{k_{1}, \ldots, k_{m}\right\}$. Set $p_{0}=$ $\max \left\{r_{k_{1}}, \ldots, r_{k_{m}}\right\}+1$ and let $j \in\left\{1, \ldots, p_{0}\right\}$. If $j \in\left\{k_{1}, \ldots, k_{m}\right\}$, then $r_{j}=r_{k_{i}}<p_{0}$. If $j \notin\left\{k_{1}, \ldots, k_{m}\right\}$, then $r_{j}<j \leq p_{0}$. Hence, for each $j \in\left\{1, \ldots, p_{0}\right\}, r_{j} \in\left\{1, \ldots, p_{0}-1\right\}$, which shows that $\left(r_{n}\right)$ cannot be one-to-one.

By our assumptions, there exists $n_{0}$ such that, for any $n \geq n_{0}, \varphi\left(\operatorname{diam}\left(I_{n}\right)\right)<$ $\operatorname{diam}\left(J_{n}\right)$. Hence, for infinitely many $n \in \mathbb{N}$,

$$
\operatorname{diam}\left(f_{n}\left(I_{r_{n}}\right)\right) \leq \operatorname{diam}\left(f_{n}\left(I_{n}\right)\right) \leq \varphi\left(\operatorname{diam}\left(I_{n}\right)\right)<\operatorname{diam}\left(J_{n}\right) .
$$

Hence $f(I)=P_{1} \times P_{2} \times P_{3} \times \cdots$, where each $P_{n}$ is an interval and, for infinitely many $n, P_{n} \neq J_{n}$. The result follows.

Case 2. Assume that $r_{n}=r_{m}$ for some $m, n$ with $m<n$. It is enough to observe that the set

$$
B=\left\{\left(f_{m}(x), f_{n}(x)\right): x \in I_{r_{n}}\right\}
$$

has an empty interior (as a subset of $J_{m} \times J_{n}$ ). Indeed, assume that this is the case and take any base open set $U=U_{1} \times U_{2} \times \cdots \subset I$. If $U \subset f(I)$, then $U_{m} \times U_{n} \subset B$, a contradiction. Hence $f(I)$ has an empty interior. Since it is also closed in $J$ (because $f(I)$ and $J$ are compact in $X)$, it is nowhere dense in $X$.

To see that $B$ has an empty interior it is enough to show that it is a subset of $\mathbb{R}^{2}$ with Lebesgue measure zero. But this follows from the fact that $B$ can be viewed as an image of a measure-zero set $I_{r_{n}} \times\{0\} \subset \mathbb{R}^{2}$ under a Lipschitz function $(x, y) \mapsto\left(f_{n}(x), f_{m}(x)\right)$. The result follows.

We are ready to give the proof of Theorem 4.3.

Proof of Theorem 4.3. Let $\phi$ be as in Lemma 4.5 and define $f_{1}, f_{2}: X \times X \rightarrow X$ in the following way:

$$
\begin{gathered}
f_{1}\left(\left(x_{n}\right),\left(y_{n}\right)\right)=\left(\phi\left(y_{1}\right), \phi\left(x_{1}\right), \phi\left(x_{2}\right), \phi\left(x_{3}\right), \ldots\right), \\
f_{2}\left(\left(x_{n}\right),\left(y_{n}\right)\right)=\left(\phi\left(y_{1}\right)+1-\phi(1), \phi\left(x_{1}\right), \phi\left(x_{2}\right), \phi\left(x_{3}\right), \ldots\right) .
\end{gathered}
$$


Then $\mathcal{S}=\left\{f_{1}, f_{2}\right\}$ is a nice GIFS. Indeed, for any $\left(\left(x_{n}\right),\left(y_{n}\right)\right),\left(\left(x_{n}^{\prime}\right),\left(y_{n}^{\prime}\right)\right) \in X \times X$, by Lemma 4.4(b),

$$
\begin{aligned}
& \left\|f_{1}\left(\left(x_{n}\right),\left(y_{n}\right)\right)-f_{1}\left(\left(x_{n}^{\prime}\right),\left(y_{n}^{\prime}\right)\right)\right\| \\
& \quad=\sup \left\{\left|\phi\left(y_{1}\right)-\phi\left(y_{1}^{\prime}\right)\right|,\left|\phi\left(x_{1}\right)-\phi\left(x_{1}^{\prime}\right)\right|,\left|\phi\left(x_{2}\right)-\phi\left(x_{2}^{\prime}\right)\right|,\left|\phi\left(x_{3}\right)-\phi\left(x_{3}^{\prime}\right)\right|, \ldots\right\} \\
& \quad \leq \sup \left\{\phi\left(\left|y_{1}-y_{1}^{\prime}\right|\right), \phi\left(\left|x_{1}-x_{1}^{\prime}\right|\right), \phi\left(\left|x_{2}-x_{2}^{\prime}\right|\right), \phi\left(\left|x_{3}-x_{3}^{\prime}\right|\right), \ldots\right\} \\
& \quad \leq \phi\left(\max \left\{\left\|\left(x_{n}\right)-\left(x_{n}^{\prime}\right)\right\|,\left\|\left(y_{n}\right)-\left(y_{n}^{\prime}\right)\right\|\right\}\right),
\end{aligned}
$$

which shows that $f_{1}$ is a $\phi$-contraction. In a similar way we can show that $f_{2}$ is a $\phi$-contraction. Hence $\mathcal{S}$ is a GIFS. Clearly, $\mathcal{S}$ is nice.

Now consider the set

$$
A=[0,1] \times[0, \phi(1)] \times\left[0, \phi^{(2)}(1)\right] \times \cdots=\prod_{n=0}^{\infty}\left[0, \phi^{(n)}(1)\right] .
$$

It turns out that $A$ is the attractor of $\mathcal{S}$. Indeed, $A$ is compact and

$$
f_{1}(A \times A)=[0, \phi(1)] \times[0, \phi(1)] \times\left[0, \phi^{(2)}(1)\right] \times \cdots
$$

and

$$
f_{1}(A \times A)=[1-\phi(1), 1] \times[0, \phi(1)] \times\left[0, \phi^{(2)}(1)\right] \times \cdots .
$$

Hence, by Lemma 4.5(d) (used for $n=0), A=f_{1}(A \times A) \cup f_{2}(A \times A)$. This gives (i).

Note that $A$ cannot be an attractor of an IFS consisting of finitely many Banach contractions since $A$ has infinite Hausdorff dimension. Indeed, by Lemma 4.5(d),

$$
\prod_{n=0}^{\infty}\left[0, \frac{1}{2^{n}}\right] \subset A
$$

and this first set has infinite Hausdorff dimension (see [MM2, Example 4.3] for details). This gives (ii).

Now let $\mathcal{S}^{\prime}=\left(g_{1}, \ldots, g_{k}\right)$ be a nice IFS consisting of $\varphi$-contractions (with the same function $\varphi$ ). In particular, for every $n \in \mathbb{N}$,

$$
\varphi\left(\operatorname{diam}\left(\left[0, \phi^{(n)}(1)\right]\right)\right)=\varphi\left(\phi^{(n)}(1)\right)<\phi^{(n)}(1)=\operatorname{diam}\left(\left[0, \phi^{(n)}(1)\right]\right) .
$$

Hence, by Lemma 4.6 (used for $I=J=A$ ), if $F_{\mathcal{S}^{\prime}}(A) \subset A$, then for each $i \in\{1, \ldots, k\}$, the set $g_{i}(A)$ is nowhere dense in $A$. Hence $F_{\mathcal{S}^{\prime}}(A)$ is nowhere dense in $A$, and, in particular, $F_{\mathcal{S}^{\prime}}(A) \neq A$. This concludes (iii).

Now let $\mathcal{S}^{\prime}=\left(g_{1}, \ldots, g_{k}\right)$ be a nice GcIFS of order $m$, and assume that $F_{\mathcal{S}^{\prime}}(A) \subset A$. We will show that, for each $i=1, \ldots, k, g_{i}(A \times \cdots \times A)$ is nowhere dense in $A$. Let $i \in\{1, \ldots, k\}$. Consider the mapping $\tilde{g}: X \rightarrow X$ given by

$$
\tilde{g}\left(\left(x_{n}\right)\right)=g_{i}\left(\left(x_{1}, x_{m+1}, x_{2 m+1}, \ldots\right),\left(x_{2}, x_{m+2}, x_{3 m+2}, \ldots\right), \ldots,\left(x_{m}, x_{2 m}, x_{3 m}, \ldots\right)\right) .
$$


Then for some sequence of naturals $\left(r_{n}\right)$,

$$
\tilde{g}_{i}\left(\left(x_{n}\right)\right)=\left(g_{1}\left(x_{r_{1}}\right), g_{2}\left(x_{r_{2}}\right), g_{3}\left(x_{r_{3}}\right), \ldots\right) .
$$

Moreover, since $\mathcal{S}^{\prime}$ is a $\operatorname{GcIFS}, \alpha:=\operatorname{Lip}\left(g_{i}\right)=\operatorname{Lip}(\tilde{g})<1$. In particular, $\tilde{g}$ is a $\varphi$ contraction with a function $\varphi(t)=\alpha t$, and

$$
\tilde{g}\left(I_{1} \times I_{2} \times I_{3} \times \cdots\right) \subset[0,1] \times[0, \phi(1)] \times\left[0, \phi^{(2)}(1)\right] \times \cdots,
$$

where $I_{m(n-1)+i}=\left[0, \phi^{(n-1)}(1)\right]$ for any $n \geq 1$ and $i \in\{1, \ldots, m\}$. By Lemma 4.5(c) and the fact that, for $n \geq 2$,

$$
\frac{\phi^{(m n)}(1)}{\phi^{(n-1)}(1)}=\frac{\phi^{(m n)}(1)}{\phi^{(n)}(1)} \frac{\phi^{(n)}(1)}{\phi^{(n-1)}(1)} \geq \frac{\phi^{(m n)}(1)}{\phi^{(n)}(1)} \frac{\phi^{(2(n-1))}(1)}{\phi^{(n-1)}(1)},
$$

there exists $n_{0} \in \mathbb{N}$ such that, for $n \geq n_{0}, \phi^{(m n)}(1)>\alpha \phi^{(n-1)}(1)$. Let $p>\left(n_{0}-1\right) m$. Then $p=(n-1) m+i$ for some $n \geq n_{0}$ and $i \in\{1, \ldots, m\}$. Thus

$$
\begin{aligned}
\varphi\left(\operatorname{diam}\left(I_{p}\right)\right) & =\varphi\left(\operatorname{diam} I_{m(n-1)+i}\right)=\varphi\left(\operatorname{diam}\left(\left[0, \phi^{(n-1)}(1)\right]\right)\right) \\
& =\alpha \operatorname{diam}\left(\left[0, \phi^{(n-1)}(1)\right]\right)<\operatorname{diam}\left(\left[0, \phi^{(m n)}(1)\right]\right) \\
& \leq \operatorname{diam}\left(\left[0, \phi^{(m(n-1)+i)}(1)\right]\right)=\operatorname{diam}\left(I_{p}\right) .
\end{aligned}
$$

Hence, by Lemma 4.5, $\tilde{g}(I)$ is nowhere dense in $A$. Since $\tilde{g}(I)=g_{i}(A \times \cdots \times A)$, we have that $g_{i}(A \times \cdots \times A)$ is nowhere dense in $A$. The proof is complete.

We believe that Lemma 4.6 is true for any IFS and GcIFS, so we state the following conjecture.

Conjecture 4.7. The set $A$, considered in the proof of Theorem 4.3, is an attractor of no IFS and of no GcIFS.

\section{Porosity results}

In this section we investigate the size of the families of all GIFSs and GcIFSs. It turns out that a natural setting for such an investigation has already been considered. In [DM, RZ1, RZ2] (and many other papers) the authors considered the problem of the size of the set of all mappings which satisfy some fixed point property, in the space of all nonexpansive(-type) mappings. In particular, it was proved that if $K$ is a nonempty closed convex and bounded subset of a Banach space, then the complement of the set of all nonexpansive selfmappings of $K$ which satisfy the hypothesis of the Banach fixed point theorem is a $\sigma$-lower porous subset of the space $\Omega$ of all nonexpansive selfmappings of $K$, and that the set of all Banach contractions is a $\sigma$-lower porous subset of $\Omega$, provided that $K$ is a subset of a Hilbert space. In [S1] we generalise these results by considering more general spaces. These generalisations can be applied for investigating the size of the families of all GIFSs and GcIFSs. 
We now present these results (in a slightly less general form than we did in [S1]). If $(X, d)$ and $(Y, \rho)$ are metric spaces, then we define:

$\boldsymbol{\Omega}(X, Y):=\left\{f: X \rightarrow Y: f(X)\right.$ is bounded and $\left.\forall_{x, y \in X} \rho(f(x), f(y)) \leq d(x, y)\right\}$.

We can consider $\boldsymbol{\Omega}(X, Y)$ as a metric space with a standard supremum metric:

$$
h(f, g):=\sup \{\rho(f(x), g(x)): x \in X\},
$$

which is complete provided that $Y$ is complete.

Define the following family of functions:

$$
\mathbf{R a}:=\{\eta:[0, \infty) \rightarrow[0,1]: \eta \text { is nonincreasing and } \eta(t)<1 \text { for } t>0\} .
$$

Define also the following subsets of $\boldsymbol{\Omega}(X, Y)$ :

$$
\mathbf{k B}(X, Y):=\{f \in \mathbf{\Omega}(X, Y): \operatorname{Lip}(f)<1\}
$$

and

$$
\mathbf{k R}(X, Y):=\left\{f \in \mathbf{\Omega}(X, Y): \exists_{\eta \in \mathbf{R a}} \forall_{x, y \in X} \rho(f(x), f(y)) \leq \eta(d(x, y)) d(x, y)\right\} .
$$

Remark 5.1. It is known (see [J, Lemma 1]) that that for any $\eta \in \mathbf{R a}$, there exists an upper semicontinuous, nondecreasing function $\varphi:[0, \infty) \rightarrow[0, \infty)$ with $\varphi(t)<t$ for $t>0$, and such that $\eta(t) \leq \varphi(t)$ for $t \geq 0$. (For detailed discussion of different types of contractive conditions we refer the reader to [J].) Hence each element of $\mathbf{k} \mathbf{R}(X, Y)$ is a generalised $\varphi$-contraction.

The following result follows from our previous research. Part (i) is implied by [S1, Theorem 3.1.22, Corollary 3.1.30 and Remark 3.1.21] (see also [S3]), and part (ii) is exactly [S2, Corollary 3.2(i)] (see also [S1, Theorem 3.2.7(i)]).

Theorem 5.2. Assume that $X, Y$ are nonempty convex and closed subsets of (possibly different) Banach spaces. The following conditions hold.

(i) The set $\mathbf{\Omega}(X, Y) \backslash \mathbf{k} \mathbf{R}(X, Y)$ is a $\sigma$-lower porous subset of $\mathbf{\Omega}(X, Y)$.

(ii) If additionally $X, Y$ are subsets of (possibly different) Hilbert spaces, then the set $\mathbf{k B}(X, Y)$ is a $\sigma$-lower porous subset of $\mathbf{\Omega}(X, Y)$.

In view of the above considerations it is natural to consider GIFSs of order $m$ and of 'length' $n$ as a subset of the following space (here, $(X, d)$ is a metric space):

$$
\mathbf{\Omega}_{m, n}(X):=\left\{\left(f_{1}, \ldots, f_{n}\right): f_{i} \in \mathbf{\Omega}\left(X^{m}, X\right)\right\}=\mathbf{\Omega}\left(X^{m}, X\right) \times \cdots \times \mathbf{\Omega}\left(X^{m}, X\right) .
$$

Then we define

$$
\operatorname{GIFS}_{m, n}^{b}(X):=\left\{\left(f_{1}, \ldots, f_{n}\right) \in \mathbf{\Omega}_{m, n}(X):\left(f_{i}\right)_{i=1}^{n} \text { is a GIFS }\right\}
$$

and

$$
\operatorname{GcIFS}_{m, n}^{b}(X):=\left\{\left(f_{1}, \ldots, f_{n}\right) \in \mathbf{\Omega}_{m, n}(X):\left(f_{i}\right)_{i=1}^{n} \text { is a GcIFS }\right\}
$$


Note that the superscript index $b$ is connected to the fact that we restricted our definition to those $f_{i}$, which have bounded images.

Since each $n$-tuple of functions $\left(f_{1}, \ldots, f_{n}\right)$ is a function from $X^{m}$ to $X^{n}$, and each function $f: X^{m} \rightarrow X^{n}$ is an $n$-tuple $f=\left(f_{1}, \ldots, f_{n}\right)$ for some $f_{1}, \ldots, f_{n}$, we can easily see that $\boldsymbol{\Omega}_{m, n}(X)=\mathbf{\Omega}\left(X^{m}, X^{n}\right), \operatorname{GcIFS}_{m, n}^{b}(X)=\mathbf{k B}\left(X^{m}, X^{n}\right)$ and, in view of Remark 5.1, $\mathbf{k R}\left(X^{m}, X^{n}\right) \subset \operatorname{GIFS}_{m, n}^{b}(X)$. Therefore, applying Theorem 5.2, we get the following result.

Theorem 5.3. Assume that $X$ is a nonempty convex and closed subset of Banach space and $m, n \in \mathbb{N}$. Then the set $\boldsymbol{\Omega}_{m, n}(X) \backslash \mathrm{GIFS}_{m, n}^{b}(X)$ is a $\sigma$-lower porous subset of $\boldsymbol{\Omega}_{m, n}(X)$. In particular, the set of all $n$-tuples $\left(f_{1}, \ldots, f_{n}\right)$ from $\boldsymbol{\Omega}_{m, n}(X)$, such that the function $F_{\mathcal{S}}$ defined as in (2.2) (for $\left.\mathcal{S}:=\left(f_{i}\right)_{i=1}^{n}\right)$ generates the fractal in the sense of Theorem 3.11, is a complement of a $\sigma$-lower porous subset of $\mathbf{\Omega}_{m, n}(X)$.

THeOrem 5.4. If $X$ is a nonempty, convex and closed subset of a Hilbert space, then the set $\mathrm{GcIFS}_{m, n}^{b}(X)$ is a $\sigma$-lower porous subset of $\mathbf{\Omega}_{m, n}(X)$.

\section{References}

[B] M. F. Barnsley, Fractals Everywhere (Academic Press, Boston, 1993).

[Br] F. Browder, 'On the convergence of successive approximations for nonlinear functional equations', Indag. Math. 30 (1968), 27-35.

[DM] F. De Blasi and J. Myjak, 'Sur la porosité de l'ensemble des contractions sans point fixe', C. $R$. Acad. Sci. Paris 308 (1989), 51-54.

[GD] A. Granas and J. Dugundji, Fixed Point Theory, Springer Monographs in Mathematics (Springer, New York, 2003).

[H] J. Hutchinson, 'Fractals and self-similarity', Indiana Univ. Math. J. 30(5) (1981), 713-747.

[J] J. Jachymski and I. Jóźwik, 'Nonlinear contractive conditions: a comparison and related problems', Banach Center Publ. 77 (2007), 123-146.

[M] A. Mihail, 'Recurrent iterated function systems', Rev. Roumaine Math. Pures Appl. 53(1) (2008), 43-53.

[Ma] L. Máté, 'The Hutchinson-Barnsley theorey for certain noncontraction mappings', Period. Math. Hungar. 27(1) (1993), 21-33.

[MM1] A. Mihalil and R. Miculescu, 'Applications of fixed point theorems in the theory of generalized IFS', Fixed Point Theory and Applications 2008 (2008), article ID 312876, 11 pp; doi: $10.1155 / 2008 / 312876$.

[MM2] A. Michalil and R. Miculescu, 'Generalized IFSs on noncompact spaces', Fixed Point Theory and Applications 2010 (2010), article ID 584215, 11 pp; doi:10.1155/2010/584215.

[S1] F. Strobin, Genericity and Porosity of Some Subsets of Function Spaces, Doctorial Dissertation, Polish Academy of Sciences, 2011.

[S2] F. Strobin, 'Some porous and meager sets of continuous mappings', J. Nonlinear Convex Anal. 13(2) (2012), 351-361.

[S3] F. Strobin, ' $\sigma$-porous sets of generalized nonexpansive mappings', Fixed Point Theory, to appear.

[Se] M. Serban, 'Fixed point theorems for operators on Cartesian product spaces and applications', Semin. Fixed Point Theory Cluj-Napoca 3 (2002), 163-172.

[RZ1] S. Reich and A. Zaslavski, 'Almost all nonexpansive mappings are contractive', C. R. Math. Acad. Sci. Soc. R. Can. 22(3) (2000), 118-124.

[RZ2] S. Reich and A. Zaslavski, 'The set of noncontractive mappings is $\sigma$-porous in the space of all nonexpansive mappings', C. R. Acad. Sci. Paris Sér. I Math. 333(6) (2001), 539-544. 
[Z1] L. Zajíček, 'Porosity and $\sigma$-porosity', Real Anal. Exchange 13(2) (1987/88), 314-350.

[Z2] L. Zajičcek, 'On $\sigma$-porous sets in abstract spaces', Abstr. Appl. Anal. 5 (2005), 509-534.

FILIP STROBIN, Institute of Mathematics, Łódź University of Technology, Wólczańska 215, 93-005 Łódź, Poland e-mail: filip.strobin@p.lodz.pl

JAROSŁAW SWACZYNA, Institute of Mathematics, Łódź University of Technology, Wólczańska 215, 93-005 Łódź, Poland e-mail: jswaczyna@wp.pl 